Pure and Applied Mathematics Quarterly

Volume 9, Number 3

$437-459,2013$

\title{
Boltje-Maisch Resolutions of Specht Modules
}

\author{
Xingyu Dai, Fang Li* and Kefeng Liu
}

\begin{abstract}
In [5], Boltje and Maisch found a permutation complex of Specht modules in representation theory of Hecke algebras, which is the same as the Boltje-Hartmann complex appeared in the representation theory of symmetric groups and general linear groups. In this paper we prove the exactness of Boltje-Maisch complex in the dominant weight case.

Keywords: Specht Module, Boltje-Maisch complex, Hecke algebra, Kempf vanishing theorem, Woodcock condition.
\end{abstract}

\section{Introduction}

In the module category of the group algebra $R \mathfrak{S}_{r}$ over an arbitrary commutative ring $R$, Boltje and Hartmann constructed a finite chain complex in [4] for any composition $\lambda$ of a positive integer $r$. Almost all factors are constructed by restricted subsets of homomorphisms between permutation modules except the last one, which is the dual of Specht module $S^{\lambda}$. Partial exactness results of position -1 and 0 about this complex were already achieved in Boltje and Hartmann's work [4] and a full proof of the exactness was obtained recently in [25] with help of Bar resolution in homology theory and the construction using the Schur functor. In [1] [2] [3] [8] [9] [24] [26] [30] [27] [31] and etc., some other permutation resolutions of Specht modules have been established.

In [5], the construction of the chain complex of $R \mathfrak{S}_{r}$ was lifted to a chain complex of modules of the Iwahori-Hecke algebra $\mathcal{H}_{r}$ with an integral domain

Received December 27, 2012.

2010 Mathematics Subject Classification: 20G43.

*The corresponding author. 
$R$ and the original chain complex reproduced by the specialization $q=1$ and moreover. This work and its method are useful for our discussion here.

The construction of this complex was completely combinatorial and characteristic free. Some partial exactness of this complex has been found in [5]. In this paper, motivated by the conclusion in [5], we find a way to prove that that this chain complex is exact whenever $\lambda$ is a partition. In order to prove its exactness in the dominant weight case, we follow the method of [25], which constructs a bar resolution in the Borel subalgebra case and transformed it into the module category of $q$-Schur algebras by induced Functors.

The paper is organized as follows. In section 2 , we find an ideal sequence $J_{0} \supseteq J_{1} \supseteq \cdots \supseteq 0$, and use $J_{0}$ and $J_{1}$ to construct a bar resolution of the module in the representations of Borel subalgebra $S_{R}^{+}(n, r)$, just as Ana Paula Santana and Ivan Yudin did in the case of symmetric groups, as in [25]. However, since the proof of vanishing theorem in [27] failed for the case of Iwahori Hecke algebra's. In section 3 , we use different tools to prove the module $R_{\lambda}$ is $S_{R}(n, r) \otimes_{S_{R}^{+}(n, r)^{-}}$ acyclic, which was introduced in [29]. After that, we reach the main results Theorem 4.7 and Theorem 5.3 which give the positive answer of exactness of Boltje-Maisch given in [5].

\section{Notations and Quoted Results}

\subsection{Combinatorics. [25]}

For any natural number $n$, we denote by $\bar{n}$ the set $\{1, \ldots, n\}$. Given a finite set $X$, for each map $\mu$ from $X$ to no negative integers $\mathbb{N}_{0}$, we can define its length $|\mu|:=\sum_{x \in X} \mu_{x}$, where we realize the map $\mu$ as $\mu(x)=\mu_{x}$ for any $x \in X$. Then, we can write a subset $\Lambda(X ; r)$ as $\left\{\mu: X \rightarrow \mathbb{N}_{0}|| \mu \mid=r\right\}$, and get a map from the set $X^{r}$ to $\Lambda(X ; r)$ as following:

$$
\begin{aligned}
w t: X^{r} & \rightarrow \Lambda(X ; r) \\
\text { with } w t(u)_{x} & :=\#\left\{s \mid u_{s}=x, s=1, \ldots, r\right\} .
\end{aligned}
$$

Here we give some notations for convenience. First, we denote $I(n, r)$ as the set $\bar{n}^{r}$. The elements of $I(n, r)$ are usually called multi-indices in other's work as [25], which can be denoted by bold letters such as $\mathbf{i}$, where $\mathbf{i}:=\left(i_{1}, \cdots, i_{r}\right)$ for $1 \leq i_{k} \leq n$ and $1 \leq k \leq r$. Also, we can denote the set $\bar{n} \times \bar{n}$ alternatively 
as $\{(i, j) \mid 1 \leq i, j \leq n\}$. Similarly, we can identify the set $(\bar{n} \times \bar{n})^{r}$ and $I(n, r) \times$ $I(n, r)$ via the map $\left(\left(i_{1}, j_{1}\right), \ldots,\left(i_{r}, j_{r}\right)\right) \mapsto\left(\left(i_{1}, \ldots, i_{r}\right),\left(j_{1}, \ldots, j_{r}\right)\right)$ without any confusions.

For convenience, from now on sets $\Lambda(\bar{n} ; r)$ and $\Lambda(\bar{n} \times \bar{n} ; r)$ are denoted by $\Lambda(n, r)$ and $\Lambda(n, n ; r)$, respectively. We can notice that the elements of $\Lambda(n, r)$ are the same as compositions of $r$ into $n$ parts, which also has same notation as $\Lambda(n, r)$ in many works as in [22]. On the set $I(n, r)$ we defined the ordering $\leq$ by

$$
\mathbf{i} \leq \mathbf{j} \Longleftrightarrow i_{1} \leq j_{1}, i_{2} \leq j_{2}, \ldots, i_{r} \leq j_{r}
$$

and write $\mathbf{i}<\mathbf{j}$ if $\mathbf{i} \leq \mathbf{j}$ and $\mathbf{i} \neq \mathbf{j}$.

2.2. Symmetric groups and Iwahori-Hecke algebras. A composition $\lambda$ of $r$ is a finite sequence of non-negative integers $\left(\lambda_{1}, \lambda_{2}, \ldots, \lambda_{n}\right)$ such that $|\lambda|=$ $\sum_{i} \lambda_{i}=r$. Moreover, there is a partial order $\unlhd($ resp. $\unrhd)$ within compositions of $r$ as: we denote $\lambda \unlhd \mu$ when $\sum_{i=1}^{k} \lambda_{i} \leq \sum_{i=1}^{k} \mu_{i}$ (resp. $\left.\sum_{i=1}^{k} \lambda_{i} \geq \sum_{i=1}^{k} \mu_{i}\right)$ for all $1 \leq k \leq n$.

Let $\mathfrak{S}_{r}$ denote the symmetric group of all permutations of $1, \ldots, r$ with Coxeter generators $s_{i}:=(i, i+1)$, and $\mathfrak{S}_{\lambda}$ the Young subgroup corresponding to the composition $\lambda$ of $r$. Thus, we have

$$
\mathfrak{S}_{\lambda}=\mathfrak{S}_{\mathbf{a}}=\mathfrak{S}_{\left\{1, \ldots, a_{1}\right\}} \times \mathfrak{S}_{\left\{a_{1}+1, \ldots, a_{2}\right\}} \times \cdots \times \mathfrak{S}_{\left\{a_{n-1}+1, \ldots, a_{n}\right\}},
$$

where $\mathbf{a}=\left[a_{0}, a_{1}, \ldots, a_{n}\right]$ with $a_{0}=0$ and $a_{i}=\lambda_{1}+\cdots+\lambda_{i}$ for all $i=1, \ldots, n$. We denote by $\mathscr{D}_{\lambda}$ the set of distinguished representatives of right $\mathfrak{S}_{\lambda}$-cosets and write $\mathscr{D}_{\lambda \mu}:=\mathscr{D}_{\lambda} \cap \mathscr{D}_{\mu}^{-1}$, which is the set of distinguished representatives of double cosets $\mathfrak{S}_{\lambda} \backslash \mathfrak{S}_{r} / \mathfrak{S}_{\mu}$.

As usual one identifies the composition $\lambda$ with Young diagram and we say that $\lambda$ is the shape of the corresponding Young diagram. For example, we can represent the partition $(3,2)$ as $\square$. A $\lambda$-tableau is a filling of the $r$ boxes of the Young diagram of $\lambda$ of the numbers $1,2, \ldots, r$. We denote the set of $\lambda$-tableaux by $\mathcal{T}(\lambda)$ and usually denote an element of $\mathcal{T}(\lambda)$ as $\mathfrak{t}$.

The group $\mathfrak{S}_{r}$ acts from the right on $\mathcal{T}(\lambda)$ by simply applying an element $w \in \mathfrak{S}_{r}$ to the entries of the tableau $\mathfrak{t} \in \mathcal{T}(\lambda)$. This action is free and transitive, and it yields a bijection

$$
\mathfrak{S}_{r} \stackrel{\sim}{\rightarrow} \mathcal{T}(\lambda), \quad w \mapsto t^{\lambda} w .
$$


A $\lambda$-tableau $\mathfrak{t}$ is called row-standard if its entries are increasing in each row from left to right. The row-standard tableaux form a subset $\mathcal{T}^{r s}(\lambda)$ of $\mathcal{T}(\lambda)$. Two $\lambda$-tableaux $\mathfrak{t}_{1}$ and $\mathfrak{t}_{2}$ are called row-equivalent if $\mathfrak{t}_{1}$ and $\mathfrak{t}_{2}$ can arise from each other by rearranging elements within each row. We denote the row-equivalent class of $\mathfrak{t}$ by $\{\mathfrak{t}\}$ and the set of row equivalent classes by $\overline{\mathcal{T}}(\lambda)$. One has the canonical bijections

$$
\mathscr{D}_{\lambda} \stackrel{\sim}{\rightarrow} \mathcal{T}^{r s}(\lambda) \stackrel{\sim}{\rightarrow} \overline{\mathcal{T}}(\lambda)
$$

given by $d \mapsto \mathfrak{t}^{\lambda} d$ and $\mathfrak{t} \mapsto\{\mathfrak{t}\}$.

Definition 2.1. Let $R$ be a commutative domain with 1 and let $q$ be a unitary element of $R$. The Iwahori-Hecke algebra $\mathcal{H}_{r}=\mathcal{H}_{R, q}\left(\mathfrak{S}_{r}\right)$ of $\mathfrak{S}_{r}$ is the unital associative $R$-algebra with generators $T_{1}, T_{2}, \ldots, T_{r-1}$ and relations:

$$
\begin{aligned}
\left(T_{i}-q\right)\left(T_{i}+1\right) & =0, & & \text { for } i=1,2, \ldots, r-1, \\
T_{i} T_{j} & =T_{j} T_{i}, & & \text { for } 1 \leq i<j-1 \leq r-2, \\
T_{i} T_{i+1} T_{i} & =T_{i+1} T_{i} T_{i+1}, & & \text { for } i=1,2, \ldots, r-2 .
\end{aligned}
$$

$\mathcal{H}_{r}$ is a free $R$-module with a finite $R$-basis $\left\{T_{w} \mid w \in \mathfrak{S}_{r}\right\} . T_{w}$ is defined as $T_{i_{1}} T_{i_{2}} \cdots T_{i_{s}}$ if $w$ has a reduced presentation $w=s_{i_{1}} s_{i_{2}} \cdots s_{i_{s}}$. Then, we put $x_{\mu}:=\sum_{w \in \mathfrak{S}_{\mu}} T_{w}$ for any Young subgroup $\mathfrak{S}_{\mu}$, which is an element in $\mathcal{H}_{r}$, and define $M^{\mu}$ to be the right $\mathfrak{S}_{r}$-module $x_{\mu} \mathcal{H}_{r}$.

Definition 2.2. Fix a non-negative integer $r$, the $q$-Schur algebra is the endomorphism algebra

$$
S_{R}(n, r)=\operatorname{End}_{\mathcal{H}_{r}}\left(\bigoplus_{\mu \in \Lambda(n, r)} M^{\mu}\right)=\bigoplus_{\lambda, \mu \in \Lambda(n, r)} \operatorname{Hom}_{\mathcal{H}_{r}}\left(M^{\mu}, M^{\lambda}\right)
$$

For any $\lambda, \mu \in \Lambda(n, r)$, each element $d$ of the set $\mathscr{D}_{\lambda \mu}:=\mathscr{D}_{\lambda} \cap \mathscr{D}_{\mu}^{-1}$ is a representative of the double cosets $\mathfrak{S}_{\lambda} \backslash \mathfrak{S}_{r} / \mathfrak{S}_{\mu}$. By [6], $\mathscr{D}_{\lambda \mu}$ forms an $R$-basis for $\operatorname{Hom}_{\mathcal{H}_{r}}\left(M^{\mu}, M^{\lambda}\right)$, where $\psi_{\lambda \mu}^{d}$ is defined by

$$
\psi_{\lambda \mu}^{d}\left(x_{\mu}\right)=\sum_{w \in \mathfrak{S}_{\lambda} d \mathfrak{S}_{\mu}} T_{w}=x_{\lambda} \sum_{e \in \mathcal{D}_{\nu} \cap \mathfrak{S}_{\mu}} T_{d e}=x_{\lambda} T_{d} \sum_{e \in \mathcal{D}_{\nu} \cap \mathfrak{S}_{\mu}} T_{e},
$$

for any parameter $d \in \mathscr{D}_{\lambda \mu}$, and where $\nu \in \Lambda(n, r)$ is determined by Young subgroup $\mathfrak{S}_{\nu}:=d^{-1} \mathfrak{S}_{\lambda} d \cap \mathfrak{S}_{\mu}$.

We can identify the set $\mathscr{D}_{\lambda \mu}$ with a combinatorial set $\mathcal{T}(\lambda, \mu)$, which is call a set of generalized tableaux of $\lambda$ with content $\mu$. Each element $T \in \mathcal{T}(\lambda, \mu)$ can be 
realized as Young diagram of composition $\lambda$ whose boxes are filled with $r$ positive integers where $\mu_{1}$ entries equal to $1, \mu_{2}$ entries equal to 2 , etc.

We denote $T_{\mu}^{\lambda}$ by the generalized tableau whose boxes are filled in the natural order, which is the stabilizer of left group action $(w T)(i):=T(i w)$. If two generalized tableaux $T_{1}, T_{2}$ arised form each other by rearranging the entries within the rows, We call they row-equivalent, i.e., $T_{2}=T_{1} w$ for some $w \in \mathfrak{S}_{\lambda}$. We denote the set of row equivalent classes by $\overline{\mathcal{T}}(\lambda, \mu)$. Furthermore, we can achieve a bijection $\mathfrak{S}_{\lambda} \backslash \mathfrak{S}_{r} / \mathfrak{S}_{\mu} \stackrel{\sim}{\rightarrow} \overline{\mathcal{T}}(\lambda, \mu), \mathfrak{S}_{\lambda} w \mathfrak{S}_{\mu} \mapsto w T_{\mu}^{\lambda}$.

A generalized tableau $T \in \mathcal{T}(\lambda, \mu)$ is said to be row-semistandard if its entries are in the natural order from left to right. Write by $\mathcal{T}^{r s}(\lambda, \mu)$ to denote the set of all such tableau. Every row-equivalent class possesses a unique row-semistandard element. It follows that $\mathcal{T}^{r s} \stackrel{\sim}{\longrightarrow} \overline{\mathcal{T}}(\lambda, \mu), T \mapsto\{T\}$, is a bijection. Therefore, we have reached a canonical bijection:

$$
\mathscr{D}_{\lambda \mu} \stackrel{\sim}{\rightarrow} \mathfrak{S}_{\lambda} \backslash \mathfrak{S}_{r} / \mathfrak{S}_{\mu} \stackrel{\sim}{\rightarrow} \overline{\mathcal{T}}(\lambda, \mu) \stackrel{\sim}{\leftarrow} \mathcal{T}^{r s}(\lambda, \mu)
$$

Moreover, Theorem 4.7 of [22] shows that $\operatorname{Hom}_{\mathcal{H}}\left(M^{\mu}, M^{\lambda}\right)$ is free as an $R$ module with basis $\left\{\psi_{\lambda \mu}^{d} \mid d \in \mathscr{D}_{\lambda \mu}\right\}$. The $q$-Schur algebra $S_{R}(n, r)$ also can be written as a free $R$-module $\underset{\substack{\lambda, \mu \in \Lambda(n, r) \\ d \in \mathscr{D} \lambda \mu}}{\bigoplus} R \psi_{\lambda \mu}^{d}$.

2.3. Quantized enveloping algebras. In order to show the vanishing theorem in the next section, we need some notations about quantum groups here. Recall the definition of the quantized enveloping algebra in the version given by Kashiwara.

Let $A=\left(a_{i, j}\right)_{i, j \in I}$ be a Cartan matrix of finite-type. Thus, there exists a sequence of positive integers $\left(d_{i}\right)_{i} \in I$ such that $d_{i} a_{i, j}=d_{j} a_{j, i}$ for all $i, j \in I$. In addition, we need it to satisfy the structure of root datum as in [19]:

(i) A perfect pairing $<,>: P^{*} \times P \rightarrow \mathbb{Z}$, where $P$ and $P^{*}=\operatorname{Hom}(P, \mathbb{Z})$ are finitely generated free $\mathbb{Z}$-modules.

(ii) Linearly independent subsets $\left\{\alpha_{i} \mid i \in I\right\}$ of $P$ and $\left\{\alpha_{i}^{\vee} \mid i \in I\right\}$ of $P^{+}$, satisfying $a_{i, j}=<\alpha_{i}^{\vee}, \alpha_{j}>$ for all $i$ and $j$.

$P$ and $P^{*}$ are called the lattices of weight and coweights, respectively; the $\alpha_{i}$ are the simple roots, the $\alpha_{i}^{\vee}$ are the simple coroot. The dominance order on $P$ is defined by $\lambda \geq \mu$ if and only if $\lambda-\mu$ can be written as a sum of simple roots. 
A weight $\lambda$ is dominant (resp. antidominant) if all $\left\langle\alpha_{i}^{\vee}, \lambda\right\rangle$ are nonnegative (resp. nonpositive).

Definition 2.3. Let $U$ be the $\mathbb{Q}(q)$-algebra with generators $e_{i}, f_{i}, q^{h}, 1 \leq i \leq n$, $h \in P^{*}$, and relations

$$
\begin{aligned}
q^{0} & =1, \quad q^{h} q^{h^{\prime}}=q^{h+h^{\prime}}, \\
q^{h} e_{i} & =q^{<h, \alpha_{i}>} e_{i} q^{h}, \\
q^{h} f_{i} & =q^{-<h, \alpha_{i}>} f_{i} q^{h}, \\
{\left[e_{i}, f_{j}\right] } & =\delta_{i, j} \frac{t_{i}-t_{i}^{-1}}{q_{i}-q_{i}^{-1}}, \\
\sum_{l=0}^{a}(-1)^{l} e_{i}^{(l)} e_{j} e_{i}^{(a-l)} & =\sum_{l=0}^{a}(-1)^{l} f_{i}^{(l)} f_{j} f_{i}^{(a-l)}=0,
\end{aligned}
$$

where $i \neq j, a=1-a_{i, j}$.

For convenience, we have set the following abbreviations in the above relations: $t_{i}=q^{d_{i} \alpha_{i}^{\vee}}, q_{i}=q^{d_{i}}, e_{i}^{(l)}=e_{i}^{l} /[l]_{i}^{!}, f_{i}^{(l)}=f_{i}^{l} /[l]_{i}^{!}$. The subscript $i$ in $[l]_{i}^{!}$means that the $q$ in the definition of $[l]$ ! is replaced by $q_{i}$.

Take a subset $J \subseteq I$. Set three set which consist of elements of $U$ :

$$
\begin{aligned}
& \mathscr{E}_{J}=\left\{e_{i}^{(s)} \mid s \geq 0, i \in J\right\} . \\
& \mathscr{F}_{J}=\left\{f_{i}^{(s)} \mid s \geq 0, i \in J\right\} . \\
& \mathscr{H}=\left\{q^{h},\left[\begin{array}{c}
q^{h} ; 0 \\
s
\end{array}\right]\right\} \quad \text { where } \quad\left[\begin{array}{c}
x ; c \\
t
\end{array}\right]=\prod_{s=1}^{t} \frac{x q^{(c-s+1)}-x^{-1} q^{-(c-s+1)}}{q^{s}-q^{-s}} .
\end{aligned}
$$

We write $\mathscr{A}$ for the ring $\mathbb{Z}\left[q, q^{-1}\right]$ of integral Laurent polynomials in the indeterminate $q$. Let $U_{\mathscr{A}}^{-}(J), U_{\mathscr{A}}^{+}(J)$ be the $\mathscr{A}$-subalgebras of $U$ generated respectively by $\mathscr{F}_{J}, \mathscr{E}_{J}$. Similarly, we can define these subalgebra structure over any arbitrary commutative ring $R$ and denote it by $U_{R}^{+}(J)$ or $U_{R}^{-}(J)$, if there exist a ring with homomorphism from $\mathscr{A}$ to $R$, especially $\mathbb{Q}(q)$ for example.

For convenience, we usually omit the subscript when the algebras are defined over $\mathbb{Q}(q)$, Then, we use the following abbreviations: $U_{\mathscr{A}}^{\mathfrak{b}}=U_{\mathscr{A}}^{-}(I), U_{\mathscr{A}}^{\#}=U_{\mathscr{A}}^{+}(I)$, and define $U^{\mathfrak{b}}, U^{\#}$ (resp. $U_{R}^{\mathfrak{b}}, U_{R}^{\#}$ ) similarly with the ground ring $\mathbb{Q}(q)$ (resp. $R$ ).

Write $U_{R}$-Int for the category of all integrable $U_{R}$-modules $V$. It is known as in Lusztig [20] that for each $\lambda \in P^{+}, U$-Int is semisimple with a simple modules 
$\Delta(\lambda)$, which is the unique maximal integrable quotient of the Verma module $M(\lambda)$.

There is a $\mathbb{Q}(q)$-algebra anti-automorphism $u \mapsto u^{\tau}$ of $U$ given by

$$
e_{i}^{\tau}=f_{i}, \quad f_{i}^{\tau}=e_{i}, \quad\left(q^{h}\right)^{\tau}=q^{h} .
$$

If $V \in U_{R}$-Int, its contravariant dual $V^{\circ}$ is the linear dual $\operatorname{Hom}_{R}(V, R)$, with its natural right $U_{R^{-}}$action transferred to the left via $\tau$. And, we denote some new objects in the category $U_{R^{-}}$Int as $\nabla_{R}(\lambda)=\Delta_{R}(\lambda)^{\circ}$.

\section{Coordinate ring and bar resolutions}

Definition 3.1. [11] For a commutative ring $R$, let $R\left[M_{n}(q)\right]$ be the associative algebra over $R$ generated by $X_{i j}$ with $1 \leq i, j \leq n$ such that

$$
\left\{\begin{array}{cc}
X_{i j} X_{i k}=q X_{i k} X_{i j}, & \text { if } j>k, \\
X_{j i} X_{k i}=X_{k i} X_{j i}, & \text { if } j>k, \\
X_{i j} X_{r s}=q^{-1} X_{r s} X_{i j}, & \text { if } i>r, j<s, \\
X_{i j} X_{r s}-X_{r s} X_{i j}=\left(q^{-1}-1\right) X_{i s} X_{r j}, & \text { if } i<r, j<s
\end{array}\right.
$$

As an $R$-module, $R\left[M_{n}(q)\right]$ has a basis $\left\{\prod_{i j} X_{i j}^{t_{i j}} \mid t_{i j} \in \mathbb{Z}^{+}\right\}$, where the products are formed with respect to any fixed order of the $X_{i j}$ 's. Let $A_{q}(n, r)$ be the rth homogeneous component of $R\left[M_{n}(q)\right]$. Then $A_{q}(n, r)$ has a basis

$$
\left\{X_{\lambda \mu}^{d}:=X_{\mathbf{i}_{\lambda} d, \mathbf{i}_{\mu}} \mid \lambda, \mu \in \Lambda(n, r), d \in \mathscr{D}_{\lambda \mu}\right\},
$$

where $\mathscr{D}_{\lambda \mu}$ denotes the set of distinguished representatives for $\mathfrak{S}_{\lambda} \backslash \mathfrak{S}_{r} / \mathfrak{S}_{\mu}$ (see Definition 2.2), and $X_{\mathbf{i j}}=X_{i_{1} j_{1}} X_{i_{2} j_{2}} \cdots X_{i_{r} j_{r}}$ if $\mathbf{i}=\left(i_{1}, \cdots, i_{r}\right)$ and $\mathbf{j}=\left(j_{1}, \cdots, j_{r}\right)$. Denote by $A_{q}(n, r)^{*}$ the linear dual of $A_{q}(n, r)$. Then, by [11]

$$
\varphi: \operatorname{End}_{\mathcal{H}_{r}}\left(\bigoplus_{\lambda \in \Lambda(n, r)} x_{\lambda} \mathcal{H}_{r}\right) \cong A_{q}(n, r)^{*},
$$

where the natural basis for $q$-Schur algebra End $\mathcal{H}_{\mathcal{H}_{r}}\left(\bigoplus_{\lambda \in \Lambda(n, r)} x_{\lambda} \mathcal{H}_{r}\right)$ is given as follows:

For $\lambda, \mu \in \Lambda(n, r), d \in \mathscr{D}_{\lambda \mu}$, if we identify $\psi_{\lambda \mu}^{d}$ with its images under the isomorphism above. The basis $\left\{\psi_{\lambda \mu}^{d}\right\}$ is the dual of the basis $\left\{X_{\lambda \mu}^{d}:=X_{\mathbf{i}_{\lambda} d, \mathbf{i}_{\mu}} \mid \lambda, \mu \in\right.$ $\left.\Lambda(n, r), d \in \mathscr{D}_{\lambda \mu}\right\}$ for $A_{q}(n, r)$. Moreover by [11], we have $\varphi\left(\psi_{\lambda \mu}^{d}\right)\left(X_{\rho \nu}^{d_{1}}\right)=$ $\delta_{\lambda \rho} \delta_{\mu \nu} \delta_{d, d_{1}}$. Sometimes we denote the basis $\left\{\varphi\left(\psi_{\lambda \mu}^{d}\right) \mid \lambda, \mu \in \Lambda(n, r), d \in \mathscr{D}_{\lambda \mu}\right\} \subset$ $A_{q}(n, r)^{*}$ as $\left\{\psi_{\mathbf{i}_{\lambda} d, \mathbf{i}_{\mu}} \mid \lambda, \mu \in \Lambda(n, r), d \in \mathscr{D}_{\lambda \mu}\right\}$. 


\section{Remark 3.2. Combination correspondence:}

By using the notations of section 2.1, we can identify the following three sets:

$$
\begin{aligned}
\Xi: \bigsqcup_{\lambda, \mu \in \Lambda(n ; r)} \mathscr{D}_{\lambda \mu} \rightarrow I(n, r) \times I(n, r) / & \rightarrow \Lambda(n, n ; r) \\
d \in \mathscr{D}_{\lambda \mu} \mapsto \overline{\left(\mathbf{i}_{\lambda} d, \mathbf{i}_{\mu}\right)} & \mapsto w t\left(\mathbf{i}_{\lambda} d, \mathbf{i}_{\mu}\right)
\end{aligned}
$$

where we put $I(n, r) \times I(n, r)$ as a quotient set of $I(n, r) \times I(n, r)$ with following relation

$$
(\mathbf{i}, \mathbf{j}) \sim\left(\mathbf{i}^{\prime}, \mathbf{j}^{\prime}\right) \Longleftrightarrow w t(\mathbf{i}, \mathbf{j})=w t\left(\mathbf{i}^{\prime}, \mathbf{j}^{\prime}\right)
$$

and the map $w t$ is defined in the subsection 2.1 .

Remark 3.3. The elements of $\Lambda(n, n ; r)$ can be realized as $n \times n$ matrices of non-negative integers $\left(\omega_{s t}\right)_{s, t}$ with $1 \leq s, t \leq n$ such that $\sum_{s, t=1}^{n} \omega_{s t}=r$.

Denote by $\Lambda^{s}(n, n ; r)$ the subset of $\Lambda(n, n ; r)$ as

$$
\Lambda^{s}(n, n ; r)=\left\{\omega \in \Lambda(n, n ; r) \mid \omega_{i j}=0 \forall i>j, \sum_{1 \leq k \leq l \leq n}(l-k) \omega_{k l} \geq s\right\} .
$$

Under the identification in 3.2, we simply find that

(1) $(\mathbf{i}, \mathbf{j})$ satisfies that $\mathbf{i} \geq \mathbf{j} \Longleftrightarrow$ Put $\left(\omega_{i j}\right)=w t(\mathbf{i}, \mathbf{j})$, which means $\omega_{i j}=$ $0 \forall i>j$.

(2) Put $\left(\omega_{i j}\right)=w t\left(\mathbf{i}_{\lambda} d, \mathbf{j}_{\mu}\right)$. There exists a pair $i, j$ such that $\omega_{i j} \neq 0 \Longrightarrow$ $\lambda \neq \mu$.

Thus, two definitions of subsets are given as follows:

$$
\begin{gathered}
\Omega^{\succeq 0}:=\Xi^{-1}\left(\Lambda^{0}(n, n ; r)\right)=\bigsqcup_{\lambda, \mu \in \Lambda(n, r)}\left\{d \in \mathscr{D}_{\lambda \mu} \mid \mathbf{i}_{\lambda} d \geq \mathbf{i}_{\mu}\right\}:=\bigsqcup_{\lambda, \mu \in \Lambda(n, r)} \Omega_{\lambda \mu}^{\succeq 0} . \\
\Omega^{\succeq 1}:=\Xi^{-1}\left(\Lambda^{1}(n, n ; r)\right)=\left\{d \in \Omega^{\succeq 0} \mid \lambda, \mu \in \Lambda(n, r), \lambda \neq \mu \text { and } d \neq 1\right\} .
\end{gathered}
$$

Similarly, we can define a sequence of sets as $\Omega^{\succeq} \supseteq \Omega^{\succeq 1} \supseteq \Omega^{\succeq 2} \supseteq \cdots \Omega^{\succeq n} \supseteq \cdots$, and define subspaces of $S_{R}(n, r)$ :

$$
J_{n}:=\bigoplus_{d \in \Omega \succeq n} R \psi_{\lambda \mu}^{d} \subseteq S_{R}^{+}(n, r) \quad \text { with } d \in \mathscr{D}_{\lambda \mu} .
$$

We set $S_{R}^{+}(n, r):=J_{0}$, it is a Borel subalgebra of $q$-Schur algebra. This definition is the same as [12]. $J_{1}=\bigoplus_{\psi_{\lambda \mu}^{d} \neq \psi_{\lambda \lambda}^{1}} R \psi_{\lambda \mu}^{d}$ and $J_{0} \supseteq J_{1} \supseteq J_{2} \supseteq \cdots$ 
Moreover, if we define the subsets of $\Omega^{\succeq n}$ as $\Omega_{\lambda \mu}^{\succeq n}:=\Omega^{\succeq n} \cap \mathscr{D}_{\lambda \mu}$, then, we find trivially that $\bigsqcup_{\lambda, \mu \in \Lambda(n ; r)} \Omega_{\lambda \mu}^{\succeq n}=\Omega^{\succeq n}$. Using this notations, we can state the following lemmas.

Lemma 3.4. If $d_{1} \in \Omega_{\lambda \mu}^{\succeq n}$ and $d_{2} \in \Omega_{\mu \nu}^{\succeq m}$, then $\psi_{\lambda \mu}^{d_{1}} \psi_{\mu \nu}^{d_{2}}=\sum_{d \in \Omega_{\lambda \nu}^{\searrow m+n}} a_{d} \psi_{\lambda \nu}^{d}$ for some $a_{d} \in R$.

Proof. Suppose $\lambda, \mu \in \Lambda(n, r), d \in \Omega_{\lambda \mu}^{\succeq n} \subseteq \mathscr{D}_{\lambda \mu}$. We claim that $\psi_{\lambda \mu}^{d}\left(X_{\mathrm{ij}}\right) \neq 0$ implies $(\mathbf{i}, \mathbf{j}) \in I(n, r) \times I(n, r)$ satisfies $\sum_{1 \leq l \leq k \leq n}(l-k) \omega_{l k}=\sum_{k=1}^{r}\left(i_{k}-j_{k}\right) \geq n$.

Indeed, by the definition above, we have hypothesis that $\mathbf{j}=\mathbf{i}_{\mu} w$ for some $w \in \mathfrak{S}_{r}$. If $\ell(w)=0$, i.e., $w=1$, then $\mathbf{j}=\mathbf{i}_{\mu}$ and $\mathbf{i}=\mathbf{i}_{\lambda} d$, which trivially shows $(\mathbf{i}, \mathbf{j}) \in \Omega_{\lambda \mu}^{\succeq n}$.

Assume now $\ell(w)>0$. Write $w=w^{\prime} t$ with $t=(a, a+1)$ and $\ell(w)=\ell\left(w^{\prime}\right)+1$. Then by definition of $\mathbf{i}_{\mu}$, we have $j_{a}>j_{a+1}$. If $i_{a} \leq i_{a+1}$, then by $3.1, X_{\mathbf{i j}}=$ $q X \mathbf{i} t, \mathbf{j} t=q X \mathbf{i} t, \mathbf{i}_{\mu w^{\prime}}$. By induction on the length of elements in $\mathfrak{S}_{r}, \psi_{\lambda \mu}^{d}\left(X_{\mathbf{i j}}\right)=$ $q \psi_{\lambda \mu}^{d}\left(X \mathbf{i} t, \mathbf{i}_{\mu w^{\prime}}\right) \neq 0$ shows $\left(\mathbf{i} t, \mathbf{i}_{\mu} w^{\prime}\right)$ satisfy above claim, which trivially implies $(\mathbf{i}, \mathbf{j})$ satisfies this claim too.

If $i_{a}>i_{a+1}$, then also by the relations (3.1).

$$
\begin{aligned}
X_{i_{a} j_{a}} X_{i_{a+1} j_{a+1}} & =X_{i_{a+1} j_{a+1}} X_{i_{a} j_{a}}-\left(q^{-1}-1\right) X_{i_{a+1} j_{a}} X_{i_{a} j_{a+1}} \\
& =X_{i_{a+1} j_{a+1}} X_{i_{a} j_{a}}-(1-q) X_{i_{a} j_{a+1}} X_{i_{a+1} j_{a}}
\end{aligned}
$$

Thus $\psi_{\lambda \mu}^{d}\left(X_{\mathbf{i} \mathbf{j}}\right)=\psi_{\lambda \mu}^{d}\left(X_{\mathbf{i} t, \mathbf{j} t}\right)-(1-q) \psi_{\lambda \mu}^{d}\left(X_{\mathbf{i}, \mathbf{j} t}\right) \neq 0$. We have either $\psi_{\lambda \mu}^{d}\left(X_{\mathbf{i} t, \mathbf{j} t}\right) \neq 0$ or $\psi_{\lambda \mu}^{d}\left(X_{\mathbf{i}, \mathbf{j} t}\right) \neq 0$. By induction, either $(\mathbf{i} t, \mathbf{j} t)$ or $(\mathbf{i}, \mathbf{j} t)$ satisfies the above claim, which trivially shows that $(\mathbf{i}, \mathbf{j})$ satisfies this claim too.

Using the multiplication rules in $A_{q}(n, r)^{*}$, we have following:

$$
\begin{aligned}
\psi_{\lambda \mu}^{d_{1}} \cdot \psi_{\mu \nu}^{d_{2}}\left(X_{\mathbf{i j}}\right) & =<\psi_{\lambda \mu}^{d_{1}} \otimes \psi_{\mu \nu}^{d_{2}}, \triangle\left(X_{\mathbf{i j}}\right)> \\
& =\sum_{\mathbf{k} \in I(n, r)} \psi_{\lambda \mu}^{d_{1}}\left(X_{\mathbf{i k}}\right) \psi_{\mu \nu}^{d_{2}}\left(X_{\mathbf{k j}}\right) \neq 0 .
\end{aligned}
$$

So there is $\mathbf{k} \in I(n, r)$ such that $\psi_{\lambda \mu}^{d_{1}}\left(X_{\mathbf{i k}}\right) \neq 0$ and $\psi_{\mu \nu}^{d_{2}}\left(X_{\mathbf{k j}}\right) \neq 0$. Thus, $\sum_{h=1}^{r}\left(i_{h}-\right.$ $\left.k_{h}\right) \geq n$ and $\sum_{l=1}^{r}\left(k_{l}-j_{l}\right) \geq m$, and hence $\sum_{s=1}^{r}\left(i_{s}-j_{s}\right)=\sum\left(i_{h}-k_{h}\right)-\sum\left(k_{l}-j_{l}\right) \geq$ $n+m$. 
And we only need to show that $a_{d} \neq 0$ only if $\mathbf{i}_{\lambda} \succeq \mathbf{i}_{\nu}$, which has already been done in [12] by Du and Rui.

Proposition 3.5. The subspaces $\left\{J_{m}\right\}$ with $m \in \mathbb{N}_{0}$ are ideals of $S_{R}^{+}(n, r)$. Moreover, nilpotent ideal $J_{1}$ is actually the radical of $S_{R}^{+}(n, r)$ when $R$ is a field, which is spanned by $\left\{\psi_{\lambda \mu}^{d} \mid \lambda \neq \mu, d \neq 1\right\}$.

Proof.

$$
\begin{aligned}
\psi_{\lambda \mu}^{d_{1}} \cdot \psi_{\omega \nu}^{d_{2}}\left(X_{\mathbf{i j}}\right) & =<\psi_{\lambda \mu}^{d_{1}} \otimes \psi_{\omega \nu}^{d_{2}}, \triangle\left(X_{\mathbf{i j}}\right)> \\
& =\sum_{k \in I(n, r)} \psi_{\lambda \mu}^{d_{1}}\left(X_{\mathbf{i k}}\right) \psi_{\omega \nu}^{d_{2}}\left(X_{\mathbf{k j}}\right) .
\end{aligned}
$$

Using the formula above, $\psi_{\lambda \mu}^{d}\left(X_{\rho \nu}^{d_{1}}\right)=\delta_{\lambda \rho} \delta_{\mu \nu} \delta_{d, d_{1}}$, we can claim that: if that $\mu \neq \omega$, then $\psi_{\lambda \mu}^{d_{1}} \cdot \psi_{\omega \nu}^{d_{2}}\left(X_{\mathbf{i j}}\right)=0$. Then by the consequence of Lemma 3.4, we find the subspace $J_{n}:=\bigoplus_{d \in \Omega \succeq n} R \psi_{\lambda \mu}^{d}$ is ideal of $S_{R}^{+}(n, r)$, since $J_{0} J_{n} \subseteq J_{n}$ with $J_{0}=S_{R}^{+}(n, r)$.

Let $L_{n, r}:=\bigoplus_{\lambda \in \Lambda(n, r)} R \psi_{\lambda \lambda}^{1}$, then $L_{n, r}$ is a commutative $R$-subalgebra of $S_{R}^{+}(n, r)$, and $S_{R}^{+}(n, r)=L_{n, r} \oplus J_{1}$. For every $\lambda \in \Lambda(n, r)$ we have an $R$-free module $R_{\lambda}:=R \psi_{\lambda \lambda}^{1}$ of rank one. Note that $\psi_{\lambda \lambda}^{1}$ acts on $R_{\lambda}$ as identity, and $\psi_{\mu \mu}^{1}, \mu \neq \lambda$, acts as zero. We will denote in the same way the module over $S_{R}^{+}(n, r)$ obtain from $R_{\lambda}$ by the natural projection of $S_{R}^{+}(n, r)$ on $L_{n, r}$.

Note that if $R$ is a field the algebra $L_{n, r}$ is semi-simple, and so $J_{1}$ is the radical of $S_{R}^{+}(n, r)$. In this case, $\left\{R_{\lambda} \mid \lambda \in \Lambda(n, r)\right\}$ is a complete set of pairwise non-isomorphic simple modules over $S_{R}^{+}(n, r)$.

Remark 3.6. By the result of 3.5, in the category of rings, we can construct a splitting map $p: S_{R}^{+}(n, r) \rightarrow \bigoplus_{\lambda \in \Lambda(n, r)} R \psi_{\lambda \lambda}^{1}$ of the including map $i: \bigoplus_{\lambda \in \Lambda(n, r)} R \psi_{\lambda \lambda}^{1}$ $\hookrightarrow S_{R}^{+}(n, r)$.

From now on, denote $A:=S_{R}^{+}(n, r), J:=\operatorname{rad}\left(S_{R}^{+}(n, r)\right)$ and $S:=\bigoplus_{\lambda \in \Lambda(n, r)} R \psi_{\lambda \lambda}^{1}$. Then, we define a homomorphism of $S$-bimodules as $\widetilde{p}: A \rightarrow J$ with $a \mapsto a-p(a)$. Obviously $S$ is a commutative ring and the restriction of $\widetilde{p}$ to $J$ is the identity map.

Definition 3.7. For every left $A$-module $M$, define a complex $\mathbb{C}_{*}(A, S, M)$ whose factors are denoted by $C_{k}(A, S, M)$, where integer $k \geq-1$, following the notation 
of in Remark 3.6. We set the several notations:

$$
\left\{\begin{array}{cc}
C_{-1}(A, S, M)=M & k=-1 \\
C_{0}(A, S, M)=A \otimes M & k=0 \\
C_{k}(A, S, M)=A \otimes J^{\otimes k} \otimes M & k>1
\end{array}\right.
$$

where all the tensor products are taken over $S$.

Next we define A-module homomorphism $d_{k, j}: C_{k}(A, S, M) \rightarrow C_{k-1}(A, S, M)$, $0 \leq j \leq k$, and $S$-module homomorphisms $s_{k}: C_{k}(A, S, M) \rightarrow C_{k+1}(A, S, M)$ by:

$$
d_{0,0}(m):=a m,
$$

$d_{k, 0}\left(a \otimes a_{1} \otimes \cdots \otimes a_{k} \otimes m\right):=a a_{1} \otimes a_{2} \otimes \cdots \otimes a_{k} \otimes m$,

$d_{k, j}\left(a \otimes a_{1} \otimes \cdots \otimes a_{k} \otimes m\right):=a \otimes a_{1} \otimes \cdots \otimes a_{j} a_{j+1} \otimes \cdots \otimes a_{k} \otimes m, 1 \leq j \leq k-1$,

$d_{k, k}\left(a \otimes a_{1} \otimes \cdots \otimes a_{k} \otimes m\right):=a \otimes a_{1} \otimes \cdots \otimes a_{k-1} \otimes a_{k} m$,

$$
s_{-1}(m):=e \otimes m,
$$

$s_{k}\left(a \otimes a_{1} \otimes \cdots \otimes a_{k} \otimes m\right):=e \otimes \widetilde{p}(a) \otimes a_{1} \otimes \cdots \otimes a_{k} \otimes m, 0 \leq k$.

Define an A-module homomorphism $d_{k}: C_{k}(A, S, M) \rightarrow C_{k-1}(A, S, M)$ by:

$$
d_{k}:=\sum_{t=0}^{k}(-1)^{t} d_{k, t}
$$

Proposition 3.8. [25] The sequence $\left(C_{k}(A, S, M), d_{k}\right)_{k \geq-1}$ is a complex of left A-module. Moreover, we have the relations:

$$
\begin{gathered}
d_{0} s_{-1}=i d_{C_{-1}(A, S, M)}, \\
d_{k+1} s_{k}+s_{k-1} d_{k}=i d_{C_{k}(A, S, M)}, 0 \leq k .
\end{gathered}
$$

Thus, these maps $\left\{s_{k}\right\}_{k \geq 1}$ give a splitting of $\mathbb{C}_{*}(A, S, M)$ in the category of $S$ modules, i.e,. Prove that $i d_{\mathbb{C}_{*}}$ a zero holomorphic chain map. In particular, $\left(C_{k}(A, S, M), d_{k}\right)_{k \geq-1}$ is exact.

Definition 3.9. Let $\xi$ be the anti-automorphism of the $q$-Schur algebra $S_{R}(n, r)$, which is defined as

$$
S_{R}(n, r) \rightarrow S_{R}(n, r) \quad \psi_{\lambda \mu}^{d} \mapsto \psi_{\mu \lambda}^{d^{-1}}
$$

It is clear that by definition of $S_{R}(n, r), S_{R}^{+}(n, r)$ and $S_{R}^{-}(n, r):=\xi\left(S_{R}^{+}(n, r)\right)$, this anti-automorphism $\xi$ can be restricted as a ring homomorphism $S_{R}^{+}(n, r) \rightarrow$ $S_{R}^{-}(n, r)$. Following the definition in Appendix 7 of [24], we define a functor $\mathcal{J}$ 
which is called a contravariant dual functor of the left $S_{R}(n, r)$-module category $S_{R}(n, r) \bmod$ :

$$
\begin{aligned}
S_{R}(n, r) \bmod & \rightarrow S_{R}(n, r) \bmod \\
V & \mapsto V^{\circledast}
\end{aligned}
$$

where the left module structure is defined to satisfy that $(s \theta)(v)=\theta(\xi(s) v)$, for $\theta \in H_{\text {om }}(V, R), s \in S_{R}(n, r), v \in V$.

Then we may consider the right exact functor

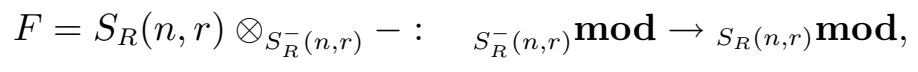

and the left exact functor

$$
G=\operatorname{Hom}_{S_{R}^{+}(n, r)}\left(S_{R}(n, r),-\right): \quad S_{R}^{+}(n, r) \bmod \rightarrow S_{R}(n, r) \bmod .
$$

Lemma 3.10. With the notation above, there is a $S_{R}(n, r)$-isomorphism

$$
F\left(V^{\circledast}\right) \cong(G(V))^{\circledast}
$$

naturally in $V \in S_{R}^{+}(n, r)$-mod

Proof. In [24](section 7), the author proved a more general result for these algebras.

Let $R_{\Lambda}:=\bigoplus_{\lambda \in \Lambda} R \psi_{\lambda}$, with $\psi_{\lambda}:=\psi_{\lambda \lambda}^{1}$. Then $R_{\Lambda}$ is a commutative $R$-subalgebra of $S_{R}^{+}(n, r)$, and $S_{R}^{+}(n, r)=R_{\Lambda} \oplus J_{1}$. For every $\lambda \in \Lambda(n, r)$ we have a free $R$-module module $L_{\lambda}:=R \psi_{\lambda}$ of rank one over $R_{\Lambda}$. Note that $\psi_{\lambda}$ acts on $L_{\lambda}$ by identity, and $\psi_{\mu}, \mu \neq \lambda$, acts by zero. We will denote in the same way the module over $S_{R}^{+}(n, r)$ obtained from $L_{\lambda}$ by inflating along the natural projection of $S_{R}^{+}(n, r)$ on $R_{\Lambda}$, i.e., $J_{1}$ and $\psi_{\mu}(\mu \neq \lambda)$ act on $L_{\lambda}$ as zero, and $\psi_{\lambda}$ as identity.

Note that if $R$ is a field then the algebra $R_{\Lambda}$ is semi-simple, and so $J_{1}$ is the radical of $S_{R}^{+}(n, r)$. In this case $\left\{L_{\lambda} \mid \lambda \in \Lambda(n, r)\right\}$ is a complete set of pairwise non-isomorphic simple modules over $S_{R}^{+}(n, r)$.

For $\lambda \in \Lambda(n, r)$ we denote the resolution $C_{*}\left(S_{R}^{+}(n, r), R_{\Lambda}, L_{\lambda}\right)$ defined in 3.7 by $\mathbb{C}_{*}^{+}\left(L_{\lambda}\right)$, and call it bar resolution. The factor $\mathbb{C}_{k}^{+}\left(L_{\lambda}\right)$ in the resolution $C_{*}\left(S_{R}^{+}(n, r), R_{\Lambda}, L_{\lambda}\right)$ has the following form:

$$
S_{R}^{+}(n, r) \otimes J_{1} \otimes \cdots \otimes J_{1} \otimes L_{\lambda},
$$


where all tensor products are over commutative ring $L_{\lambda}$ and there are $k$ factors $J_{1}$.

Proposition 3.11. Let $\nu, \mu \in \Lambda$ and $n \geq 0$. Then $\psi_{\nu} J_{n} \psi_{\mu}=0$ unless $\nu \triangleright \mu$ (which means $\nu \unrhd \mu$ but $\nu \neq \mu$ ). If $\nu \triangleright \mu$, then

$$
\left\{\psi_{\nu \mu}^{d} \mid d \in \Omega_{\nu \mu}^{\succeq n}\right\}
$$

is an $R$-basis of the free $R$-module $\psi_{\nu} J_{n} \psi_{\mu}$.

Proof. From Remark 3.3 it follow that the set $J_{n}=\bigoplus_{\substack{d \in \Omega \succeq n \\ \theta \in \eta \in \Lambda(n, r)}} R \psi_{\theta \eta}^{d}$. Then, we have

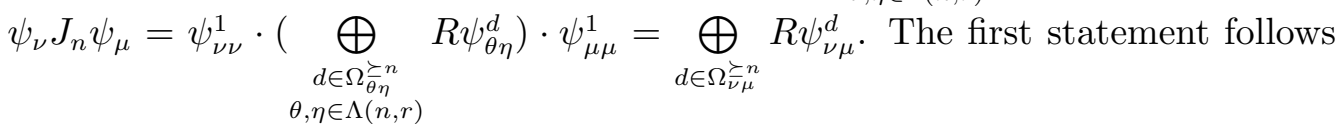
from the fact $\Omega_{\bar{\nu} \mu}^{\frac{\succeq}{\theta}} \subseteq \Omega_{\bar{\nu} \mu}^{\succ}$ and $d \in \Omega_{\bar{\nu} \mu}^{\succ}$ shows that $\nu \triangleright \mu$.

Proposition 3.12. For all $\lambda \in \Lambda(n, r)$, we have $\mathbb{C}_{0}^{+}\left(L_{\lambda}\right) \cong S_{R}^{+}(n, r) \psi_{\lambda}$, and when $k \geq 1$, factor of $\mathbb{C}_{k}^{+}\left(L_{\lambda}\right)$ has the form

$$
\bigoplus_{\mu^{(1)} \triangleright \cdots \triangleright \mu^{(k)} \triangleright \lambda} S_{R}^{+}(n, r) \psi_{\mu^{(1)}} \otimes_{R} \psi_{\mu^{(1)}} J_{1} \psi_{\mu^{(2)}} \otimes_{R} \cdots \otimes_{R} \psi_{\mu^{(k)}} J_{1} \psi_{\lambda},
$$

Proof. First of all, let $M$ be a right $R_{\Lambda}$-module and $N$ a left $R_{\Lambda}$-module. It follows from Corollary 9.3 in [21] that $M \otimes_{R_{\Lambda}} N \cong \bigoplus_{\lambda \in \Lambda(n, r)} M \psi_{\lambda} \otimes_{R} \psi_{\lambda} N$.

Then from the above statement, we can tell that $\mathbb{C}_{k}^{+}\left(L_{\lambda}\right)$ is the direct sum of $S_{R}^{+}(n, r)$-modules like:

$$
S_{R}^{+}(n, r) \psi_{\mu^{(1)}} \otimes \psi_{\mu^{(1)}} J_{1} \psi_{\mu^{(2)}} \otimes \cdots \otimes \psi_{\mu^{(k)}} J_{1} \psi_{\mu^{(k+1)}} \otimes \psi_{\mu^{(k+1)}} L_{\lambda} .
$$

where all tensor products are over $R$ and the sum is taken on any sequence $\left(\mu^{(1)}, \ldots, \mu^{(k+1)}\right) \in(\Lambda(n, r))^{k+1}$. With the property $\psi_{\mu^{(k+1)}} L_{\lambda}=0$ unless $\mu^{(k+1)}=$ $\lambda$ and the consequence of Proposition 3.12, the summation is in fact over the sequences $\mu^{(1)} \triangleright \ldots \triangleright \mu^{(k)} \triangleright \lambda$.

Proposition 3.13. Let $\lambda \in \Lambda(n, r)$. Then $\mathbb{C}_{*}^{+}\left(L_{\lambda}\right)$ is a projective resolution of the module $L_{\lambda}$ over $S_{R}^{+}(n, r)$ with finite length .

Proof. Let $N$ be the length of the maximal strictly decreasing sequence in $(\Lambda(n, r), \triangleright)$. Then $\mathbb{C}_{k}^{+}\left(L_{\lambda}\right)=0$ for $k>N$ by Proposition 3.11 and 3.12. Therefore, we can tell that $\mathbb{C}_{*}^{+}\left(L_{\lambda}\right)$ is a complex with finite length. 
The summand $S_{R}^{+}(n, r) \psi_{\mu^{(1)}} \otimes_{R} \psi_{\mu^{(1)}} J_{1} \psi_{\mu^{(2)}} \otimes_{R} \cdots \otimes_{R} \psi_{\mu^{(k)}} J_{1} \psi_{\lambda}$ of $\mathbb{C}_{*}^{+}\left(L_{\lambda}\right)$ is a projective $S_{R}^{+}(n, r)$-module, since $S_{R}^{+}(n, r) \psi_{\mu^{(1)}}$ is projective and every $\psi_{\mu^{(i)}} J_{1} \psi_{\mu^{(i+1)}}$ is isomorphic to a free $R$-module with finite rank. Then, we can say that $\mathbb{C}_{k}^{+}\left(L_{\lambda}\right)$ is a projective $S_{R}^{+}(n, r)$-module and $\mathbb{C}_{*}^{+}\left(L_{\lambda}\right)$ is a projective resolution of the module $L_{\lambda}$ over $S_{R}^{+}(n, r)$.

\section{Woodcock's condition and Kempf's Vanishing theorem}

In this section we explain a condition which appears in Kashiwara's work [18] and Woodcock's work [18], and explain how the result of Kempf's Vanishing theorem can be applied to prove that $S_{R}^{+}(n, r)$-module $L_{\lambda}$ is acyclic for the induction functor $S_{R}(n, r) \otimes_{S_{R}^{+}(n, r)}-$.

Let $P$ be a set and $\lambda \in P$, and let $R(\lambda)$ be a copy of the trivial $R$-coalgebra. Let $1_{\lambda}$ be the element of $\coprod_{\lambda \in P} R(\lambda)$ whose $\mu$-component is $\delta_{\lambda, \mu}$. Then, for any left (resp. right) comodule $(V, \rho)$ of $\coprod_{\lambda \in P} R(\lambda)$, we have $V=\otimes_{\lambda \in P}{ }^{\lambda} V$ (resp. $\left.\otimes_{\lambda \in P} V^{\lambda}\right)$, where ${ }^{\lambda} V:=\left\{v \in V \mid \rho(v)=1_{\lambda} \otimes v\right\}\left(\right.$ resp. $\left.V^{\lambda}:=\left\{v \in V \mid \rho(v)=v \otimes 1_{\lambda}\right\}\right)$ is called left (resp. right) weight space for weight $\lambda$.

Suppose we are given a partical order $\leq$ on a poset $P$ and a subset $P^{+}$of $P$ which is a locally finite poset, i.e., for each $\lambda \in P$ there are only finitely many $\mu \in P^{+}$with $\mu \leq \lambda$.

Let $\left(A(\Lambda), \mu_{\Gamma}^{\Lambda}\right)$ be a filtered system of $R$-coalgebras indexed by the finite ideals in $P^{+}$. Assume that each map $\mu_{\Gamma}^{\Lambda}: A(\Gamma) \rightarrow A(\Lambda)$ is injective, and the map $A(\Lambda) \rightarrow \coprod_{\lambda \in P} R(\lambda)$ is compatible with $\mu_{\Lambda}^{\Gamma}$. Put $C=\underset{\Lambda}{\lim } A(\Lambda)$.

Definition 4.1. Say that $\left(A(\Lambda), P^{+}, C\right)$ satisfies a Woodcock condition: if we have an isomorphism of bicomodules

$$
A(\Lambda) / A(\Lambda \backslash\{\lambda\}) \cong \nabla(\lambda) \otimes \nabla^{\prime}(\lambda) .
$$

where $\nabla(\lambda)$ and $\nabla^{\prime}(\lambda)$ are, respectively, left and right $A(\lambda)$-comodules satisfying:

(1) ${ }^{\lambda} \nabla(\lambda) \cong \nabla^{\prime}(\lambda)^{\lambda} \cong R(\lambda)$.

(2) For all $\lambda \in P,{ }^{\mu} \nabla(\lambda) \neq 0$ or $\nabla^{\prime}(\lambda)^{\mu} \neq 0$ implies $\mu \leq \lambda$.

(3) $\nabla(\lambda)$ and $\nabla^{\prime}(\lambda)$ are finitely generated and projective over $R$. 
The most valuable example of Woodcock condition appears in a quantized enveloping algebra and the coordinate algebra corresponding to it.

Example 4.2. [28] Let $U$ be a quantized enveloping algebra.

$A:=\left\{a \in U^{*} \mid U a, a U\right.$ are both integrable $\}$ has a $\mathbb{Q}(q)$-coalgebra structure where the comultiplication is defined by $\triangle(c)(u \otimes v)=c(v u)$ and the counit by $\varepsilon(c)=$ $c(1)$, for any $c \in A$ and $u, v \in U$.

In [28], Woodcock showed that, if $\Lambda$ is a finite ideal in $P^{+}$and $V \in U$-int, let $O_{\Lambda} V$ be the largest submodule $V^{\prime}$ of $V$ such that ${ }^{\lambda} V^{\prime} \neq 0, \lambda \in P^{+}$implies $\lambda \in \Lambda$. Put $A(\Lambda):=O_{\Lambda} A$, we know that $\left(A(\Lambda), P^{+}, A\right)$ is a triple which satisfies Woodcock's condition.

Remark 4.3. [28] Let $\Lambda$ be a finite ideal of $P^{+}$. Denote $A_{R}(\Lambda)=R \otimes_{\mathscr{A}} A(\Lambda)$ and $A_{R}=R \otimes_{\mathscr{A}} A$, where $A(\Lambda)$ is defined as above. Moreover, let $\lambda$ be the maximal one in $\Lambda$ and $\Gamma=\Lambda \backslash\{\lambda\}$, then we have a short exact sequence in $U$-bimodules

$$
0 \rightarrow A_{R}(\Gamma) \rightarrow A_{R}(\Lambda) \rightarrow \nabla_{R}(\lambda) \otimes \nabla_{R}(\lambda)^{\prime} \rightarrow 0,
$$

which maps those global basis elements in $A_{R}(\Lambda) \backslash A_{R}(\Gamma)$ bijectively onto the standard global basis of $\nabla(\lambda) \otimes \nabla(\lambda)^{\prime}$.

Therefore, the triple $\left(A_{R}(\lambda), P^{+}, A_{R}\right)$ satisfies the Woodcock condition defined above, where the ground ring $R$ has a ring morphism $\mathbb{Z}\left[q, q^{-1}\right] \rightarrow R$.

Definition 4.4. [18] For $\mu \in W \cdot \lambda$ with $\lambda \in P^{+}, W$ is the Weyl group associated with root datum of $U$. Let $\nu_{\mu}$ be the element of global basis of $\Delta(\lambda)$ with weight $\mu$. If $w \in W$ with $m=<\alpha^{\vee}, w \lambda>\geq 0$, we have

$$
\nu_{s_{i} w \lambda}=f_{i}^{(m)} \nu_{w \lambda}, \quad \nu_{w \lambda}=e_{i}^{(m)} \nu_{s_{i} w \lambda} .
$$

Put $\Delta^{\#}(\mu):=U^{\#} \cdot \nu_{\mu}$. They are the Demazure modules associated to $\mu$.

Example 4.5. [28] Dualizing short exact sequence (4.1) and induction on $|\Lambda|$, we give an algebra epimorphism $U_{R} \rightarrow S_{R}, u \mapsto u \cdot 1$. Write $S_{R}^{\mathfrak{b}}$ for the image of $U_{R}^{\mathfrak{b}}$ under this map.

The Borel Schur algebras still fit the framework of Woodcock's condition:

Let $\Xi \leq P$ be a finite ideal for the antipodal excellent order, then $W \Xi \cap P^{+}$ is a finite ideal in $P^{+}$. Thus we may choose $W \Xi \cap P^{+}$to be the ideal $\Lambda$. Let $F_{\Xi}: S_{R}^{b} \bmod \rightarrow S_{R}^{b} \bmod$ take $V$ to its largest quotient with weight in $\Xi$, a right exact functor. 
Put $S_{R}^{\mathfrak{b}}(\Xi)=F_{\Xi} S_{R}^{\mathfrak{b}}$. By the alternative version in [17], if $\mu$ is maximal in $\Xi$ for the antipodal excellent order, then there is a short exact sequence of bimodules

$$
0 \rightarrow \Delta_{R}^{\mathfrak{b}}(\mu) \otimes \bar{\Delta}_{R}^{\#}(\mu)^{\tau} \rightarrow S_{R}^{\mathfrak{b}}(\Xi) \rightarrow S_{R}^{\mathfrak{b}}(\Xi \backslash\{\mu\}) \rightarrow 0 .
$$

By induction on $|\Xi|$ it now can be shown that $S_{R}^{\mathfrak{b}}$ is finitely generated free over $R$. Therefore, the $A_{R}^{\mathfrak{b}}(\Xi):=\left(S_{R}^{\mathfrak{b}}(\Xi)\right)^{*}$ forms a filtered system of $R$-coalgebras. Write $C_{R}^{\mathfrak{b}}$ as the colimit of this system, which is a free $R$-coalgebra.

Suppose one takes $P$ for both $P$ and $P^{+}, \preceq$ for order $\leq$, and defines the maps $A_{R}^{\mathfrak{b}} \rightarrow \coprod_{\lambda \in P} R(\lambda)$ by using the idempotent $\varepsilon_{\mu}$. Then the triple $\left(A_{R}^{\mathfrak{b}}, P, C_{R}^{\mathfrak{b}}\right)$ satisfies the Woodcock's condition. It implies that the roles of the $\Delta(\mu)$ and $\Delta^{\prime}(\mu)$ are respectively played by $\Delta_{R}^{\mathfrak{b}}(\mu)$ and $\bar{\Delta}_{R}^{\#}(\mu)^{\prime}$.

The triple $\left(A(\Lambda), P^{+}, C\right)$ with Woodcock condition has some very interesting homological consequences [28]. One of them is called Ext-reciprocity. For $V \in$ $S(\Lambda)$-Mod, $X \in R$-Mod, and $\mu \in P$, we have

$$
\begin{array}{cc}
\operatorname{Ext}_{S(\Lambda)}^{i}\left(S(\Lambda)^{\mu} \otimes X, V\right) \cong \operatorname{Ext}_{R}^{i}\left(X,{ }^{\mu} V\right) & \forall i \geq 0, \\
\operatorname{Ext}_{S(\Lambda)}^{i}\left(V, A(\Lambda)^{\mu} \otimes X\right) \cong \operatorname{Ext}_{R}^{i}\left({ }^{\mu} V, X\right) & \forall i \geq 0 .
\end{array}
$$

A q-analogue Kempf's vanishing theorem was established by some properties of the crystal basis proved by Kashiwara in order to obtain the refined Demazure character formula in [18][23].

Woodcock used that the ideal of using the properties of the cystal basis to obtain a quantized Kempf's vanishing theorem also works for Schur algebra's version. Let us recall the Kempf's vanishing theorem in Woodcock [28]:

For any $R$-module $X$ and $\mu \in P$, note that $\mu^{+}$as the unique dominant weight in the orbit $W \mu$. We have

$$
\begin{aligned}
\operatorname{Ext}_{S_{R}^{b}}^{i}\left(S_{R}, \nabla_{R}^{\mathfrak{b}}(\mu) \otimes X\right) & \cong \operatorname{Ext}_{R}^{i}\left({ }^{\mu} S_{R}, X\right) \cong \operatorname{Ext}_{R}^{i}\left(\Delta_{R}\left(\mu^{+}\right)^{\tau}, X\right) \\
& \cong \begin{cases}\Delta_{R}\left(\mu^{+}\right) \otimes X & \text { if } i=0 \\
0 & \text { if } i>0\end{cases}
\end{aligned}
$$

Remark 4.6. The case $\mu=\mu^{+}$of 4.5 is Kempf's vanishing theorem in [23], since $H^{i}\left(U_{R} / U_{R}^{\mathfrak{b}}\right) \cong \operatorname{Ext}_{S_{R}^{b}(W \Lambda)}^{i}\left(S_{R}(W \Lambda), \Delta(\lambda)\right)$ for all $i \geq 0$. If $\Lambda$ is a finite ideal in $P^{+}$, and $V \in S_{R}^{b}(W \Lambda) \bmod$. 
Moreover, in this situation, we have $\nabla_{R}^{\mathfrak{b}}(\mu)=L_{\mu}$, and $S_{R}^{\mathfrak{b}}=S_{R}^{-}$. Thanks to Definition 4.4 of Demazure modules, which means the module $L_{\lambda}$ is $\operatorname{Hom}_{S_{R}^{-}}\left(S_{R},-\right)$ acyclic.

Theorem 4.7. For $\lambda \in \Lambda^{+}(n, r)$, the complex $S_{R}(n, r) \otimes_{S_{R}^{+}(n, r)} \mathbb{C}_{*}^{+}\left(L_{\lambda}\right)$ is a projective resolution of $W_{\lambda}^{L}:=S_{R}(n, r) \otimes_{S_{R}^{+}(n, r)} L_{\lambda}$ over $S_{R}(n, r)$.

Proof. Given $\lambda \in \Lambda^{+}(n, r)$, denote the complex $\mathbb{B}(R):=S_{R} \otimes_{S_{R}^{+}} \mathbb{C}_{*}^{+}\left(L_{\lambda}\right)$. Since the $S_{R}$-module isomorphism holds:

$$
\mathbb{B}(R) \cong \bigoplus_{\mu^{(1)} \triangleright \cdots \triangleright \mu^{(k)} \triangleright \lambda} S_{R} \psi_{\mu^{(1)}} \otimes_{R} \psi_{\mu^{(1)}} J_{1} \psi_{\mu^{(2)}} \otimes_{R} \cdots \otimes_{R} \psi_{\mu^{(k)}} J_{1} \psi_{\lambda}
$$

and $S_{R} \psi_{\mu^{(1)}}, \psi_{\mu^{(i)}} J_{1} \psi_{\mu^{(i+1)}}$ with $1 \leq i \leq k$ and $\psi_{\mu^{(k)}} J_{1} \psi_{\lambda}$ are all free $R$-modules by Proposition 3.11, it follows that all factors in $\mathbb{B}(R)$ are free $R$-modules.

The short exact sequence

$$
0 \rightarrow H_{i}(\mathbb{B}(\mathbb{Z})) \otimes R \rightarrow H_{i}(\mathbb{B}(R)) \rightarrow \operatorname{Tor}_{1}^{\mathbb{Z}}\left(H_{i-1}(\mathbb{B}(\mathbb{Z})), R\right) \rightarrow 0
$$

follows from the Universal Coefficient Theorem over the complex $\mathbb{B}(\mathbb{Z}) \otimes_{\mathbb{Z}} R \cong$ $\mathbb{B}(R)$.

Hence, in order to show that the complex $\mathbb{B}(R)$ are acyclic, it is enough to prove the claim that the complex $\mathbb{B}(\mathbb{Z})$ is acyclic.

We already know that $H_{i}(\mathbb{B}(\mathbb{Z}))$ is a finitely generated abelian group. Therefore we can write $H_{i}(\mathbb{B}(\mathbb{Z}))$ as $\mathbb{Z}^{\alpha} \bigoplus_{p \text { is a prime }}^{\bigoplus} \underset{s \geq 1}{\bigoplus}\left(\mathbb{Z} / p^{s} \mathbb{Z}\right)^{\alpha_{p s}}$, where only finitely many of the integers $\alpha, \alpha_{p s}$ are different from zero. For every prime $p$ denote by $\overline{\mathbb{F}}_{p}$ the algebraic closure of $\mathbb{F}_{p}$. We get $H_{i}(\mathbb{B}(\mathbb{Z})) \otimes_{\mathbb{Z}} \overline{\mathbb{F}}_{p} \cong \overline{\mathbb{F}}_{p}^{\sum_{s \geq 1} \alpha_{p s}}$, and also $H_{k}(\mathbb{B}(\mathbb{Z})) \otimes_{\mathbb{Z}}$ $\mathbb{Q} \cong \mathbb{Q}^{\alpha}$.

Thus, in order to prove the above claim, we only need to show that $H_{i}(\mathbb{B}(\mathbb{Z})) \otimes_{\mathbb{Z}}$ $\mathbb{Q}=0$ and $H_{i}(\mathbb{B}(\mathbb{Z})) \otimes_{\mathbb{Z}} \overline{\mathbb{F}}_{p}=0$ for any prime number $p$.

Let $\mathbb{L}$ denote either $\mathbb{Q}$ or the field $\overline{\mathbb{F}}_{p}$ for a prime $p$. Then, $H_{i}(\mathbb{B}(\mathbb{Z})) \otimes_{\mathbb{Z}} \mathbb{L}$ is a submodule of $H_{i}(\mathbb{B}(\mathbb{L}))$ due to the Universal Coefficient Theorem. Hence, in order to prove $H_{i}\left(\mathbb{B}(\mathbb{Z}) \otimes_{\mathbb{Z}} \mathbb{L}\right)=0$, it is enough to show that $H_{i}(\mathbb{B}(\mathbb{L}))=0$.

Now we prove that $H_{i}(\mathbb{B})(\mathbb{L})=0$. 
First, the algebra $S_{\mathbb{L}}(n, r)$ has an anti-involution $\xi: S_{\mathbb{L}}(n, r) \rightarrow S_{\mathbb{L}}(n, r)$ defined on the basis elements by $\varphi_{\lambda \mu}^{d} \mapsto \varphi_{\mu \lambda}^{d^{-1}}$. The image of $S_{\mathbb{L}}^{+}(n, r)$ under $\xi$ is the subalgebra $S_{\mathbb{L}}^{-}(n, r)$ of $S_{\mathbb{L}}(n, r)$.

As in Definition 3.9, this homomorphism $\xi$ induces a contravariant equivarience of the categories $\mathcal{J}:{ }_{S_{L}^{+}(n, r)} \bmod \rightarrow{ }_{S_{L}^{-}(n, r)} \bmod$.

By Lemma 3.10, the following exact functors are isomorphic:

$$
\begin{array}{r}
\mathcal{J} \circ \operatorname{Hom}_{S_{L}^{-}(n, r)}\left(S_{\mathbb{L}},-\right) \circ \mathcal{J}:{ }_{S_{L}^{+}(n, r)} \bmod \rightarrow S_{L}(n, r) \bmod \\
S_{\mathbb{L}}(n, r) \otimes_{S_{L}^{+}(n, r)}-:{ }_{S_{L}^{+}(n, r)} \bmod \rightarrow S_{L}(n, r) \\
\bmod
\end{array}
$$

Second, we recall that $\mathbb{B}(\mathbb{L})=S_{\mathbb{L}} \otimes_{S_{L}^{+}} \mathbb{C}_{*}^{+}\left(L_{\lambda}\right) \cong \mathcal{J}\left(\operatorname{Hom}_{S_{L}^{-}}\left(S_{L}, \mathcal{J}\left(B_{*}\left(L_{\lambda}\right)\right)\right)\right)$. Furthermore, by Kempf's vanishing theorem we already know that the module $L_{\lambda}$ is $\operatorname{Hom}_{S_{L}^{-}}\left(S_{\mathbb{L}},-\right)$-acyclic. Thus, after applying $\mathcal{J}$, we get that the complex $\mathbb{B}(\mathbb{L})$ is exact.

\section{The Boltje-Maisch complex}

Suppose $n \geq r$, we know that there is a partition $\delta:=(1, \ldots, 1,0, \ldots, 0) \in$ $\Lambda(n, r)$. Then there is a obvious isomorphism of algebras $\phi: \mathcal{H}_{r} \cong \psi_{\delta} S_{R}(n, r) \psi_{\delta}$. Therefore we can tell that if $M$ is an $S_{R}(n, r)$-module, the $\psi_{\delta} S_{R}(n, r) \psi_{\delta}$-module $\psi_{\delta} M$ is induced by $\phi$ which is a $R \mathcal{H}_{r}$-module too. In fact the map $M \mapsto \psi_{\delta} M$ is functorial, and it defines a functor $\mathscr{S}_{r}:_{S_{R}(n, r)} \bmod \rightarrow{ }_{R \mathcal{H}_{r}} \bmod$ which was named a Schur functor in [16].

In this section we show that for any $\lambda \in \Lambda^{+}(n, r)$ the complex $\mathscr{S}_{r}\left(S_{R} \otimes_{S_{R}^{+}}\right.$ $\left.\mathbb{C}_{*}^{+}\left(L_{\lambda}\right)\right)$ is isomorphic to the complex which has been constructed in [23]. Here, we call it a Boltje-Maisch complex.

First of all, we start with some notations and conventions.

Definition 5.1 ([4]). For any $\lambda, \mu \in \Lambda(n, r)$, there is an $R$-submodule of $\operatorname{Hom}_{\mathcal{H}}\left(M^{\mu}, M^{\lambda}\right)$, denoted by $\operatorname{Hom}_{\hat{\mathcal{H}}_{r}}\left(M^{\mu}, M^{\lambda}\right)$, which is a free R-module in the following form:

$$
H o m_{\mathcal{H}_{r}}^{\wedge}\left(M^{\mu}, M^{\lambda}\right):=\bigoplus_{d \in \Omega_{\lambda \mu}^{\succ}} R \psi_{\lambda \mu}^{d} .
$$




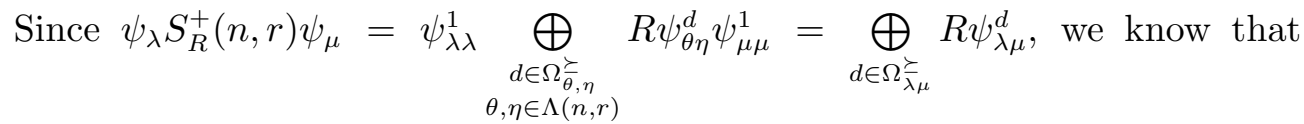
$\operatorname{Hom}_{\mathcal{H}_{r}}^{\wedge}\left(M^{\mu}, M^{\lambda}\right)$ equals $\psi_{\lambda} S_{R}^{+}(n, r) \psi_{\mu}$. Moreover, for $S_{R}^{+}(n, r)=J_{1} \oplus R_{\Lambda}$, we have $\operatorname{Hom}_{\mathcal{H}_{r}}^{\wedge}\left(M^{\mu}, M^{\lambda}\right)=\psi_{\lambda} J_{1} \psi_{\mu}$ if $\lambda \triangleright \mu$.

Boltje and Maisch defined a complex $\widetilde{B}_{*}^{\lambda}$ in Section 3.1 of [5], as follows:

For some $\lambda \in \Lambda^{+}(n, r), \widetilde{B}_{-1}^{\lambda}$ is the dual Specht module that relates to $\lambda$ and $\widetilde{B}_{0}^{\lambda}$ is defined as $\operatorname{Hom}_{R}\left(M^{\lambda}, R\right)$. When $k \geq 1, \widetilde{B}_{k}^{\lambda}$ is defined as the direct sum over all sequence $\left(\mu^{(1)} \triangleright \cdots \triangleright \mu^{(k)} \triangleright \lambda\right)$ as

$$
\begin{array}{r}
\bigoplus_{\substack{\mu^{(1)} \triangleright \ldots \triangleright \mu^{(k)} \triangleright \lambda \\
\mu^{(1)}, \ldots, \mu^{(k)} \in \Lambda^{+}(n, r)}} \operatorname{Hom}_{R}\left(M^{\mu^{(1)}}, R\right) \otimes_{R} \operatorname{Hom}_{\mathcal{H}_{r}}^{\wedge}\left(M^{\mu^{(2)}}, M^{\mu^{(1)}}\right) \otimes_{R} \\
\cdots \otimes_{R} \operatorname{Hom}_{\mathcal{\mathcal { H }}_{r}}\left(M^{\lambda}, M^{\mu^{(k)}}\right) .
\end{array}
$$

The differential $d_{k}, k \geq 1$, in $\widetilde{B}_{*}^{\lambda}$ is given by the formula

$$
d_{k}\left(f_{0} \otimes f_{1} \otimes \cdots \otimes f_{k}\right)=\sum_{t=0}^{k-1}(-1)^{t} f_{0} \otimes \cdots \otimes f_{t} \circ f_{t+1} \otimes \cdots \otimes f_{k} .
$$

and when $k=0$, we put

$$
d_{0}^{\lambda}: \widetilde{B}_{0}^{\lambda}=\operatorname{Hom}_{R}\left(M^{\lambda}, R\right) \rightarrow \operatorname{Hom}_{R}\left(S^{\lambda}, R\right)=\widetilde{B}_{-1}^{\lambda},\left.\quad \varepsilon \mapsto \varepsilon\right|_{S^{\lambda}} .
$$

and finally obtain a chain complex with only finite no trivial terms:

$$
\widetilde{B}_{*}^{\lambda}: \quad 0 \rightarrow \widetilde{B}_{f(\lambda)}^{\lambda} \stackrel{d_{f(\lambda)}^{\lambda}}{\longrightarrow} \widetilde{B}_{f(\lambda)-1}^{\lambda} \stackrel{d_{f(\lambda)-1}^{\lambda}}{\longrightarrow} \cdots \stackrel{d_{1}^{\lambda}}{\longrightarrow} \widetilde{B}_{0}^{\lambda} \stackrel{d_{0}^{\lambda}}{\longrightarrow} \widetilde{B}_{-1}^{\lambda} \rightarrow 0,
$$

where $f(\lambda)$ is a positive integer by Proposition 3.13.

According to Theorem $\mathbf{4 . 2}$ and $\mathbf{4 . 4}$ in [4], we have:

Lemma 5.2. [5] $\widetilde{B}_{*}^{\lambda}$ are exact in degree 0 and -1 .

Theorem 5.3. For $\lambda \in \Lambda^{+}(n, r)$, the complex $\widetilde{B}_{*}^{\lambda}$ is isomorphic to the complex

$$
\mathscr{S}_{r}\left(S_{R}(n, r) \otimes_{S_{R}^{+}(n, r)} \mathbb{C}_{*}^{+}\left(L_{\lambda}\right)\right) .
$$

Proof. For convenience, we denote the complex $\mathscr{S}_{r}\left(S_{R}(n, r) \otimes_{S_{R}^{+}(n, r)} \mathbb{C}_{*}^{+}\left(L_{\lambda}\right)\right)$ by $\widehat{B}_{*}^{\lambda}$. 
Since the complex $\widehat{B}_{*}^{\lambda}$ is exact, and the exactness of $\widetilde{B}_{*}^{\lambda}$ in degree 0 and -1 has been treated in Lemma 5.2, we only need to establish the isomorphism in the non-negative degrees. The isomorphism in the degree -1 will follow.

With the consequence of Proposition 3.12 , we can write the factor $\widehat{B}_{k}^{\lambda}$ as a direct sum of

$$
\psi_{\delta} S_{R}(n, r) \psi_{\mu^{(1)}} \otimes_{R} \psi_{\mu^{(1)}} J_{1} \psi_{\mu^{(2)}} \otimes_{R} \cdots \otimes_{R} \psi_{\mu^{(k)}} J_{1} \psi_{\lambda}
$$

where subscripts satisfy $\mu^{(1)} \triangleright \cdots \triangleright \mu^{(k)} \triangleright \lambda$. Furthermore, it is straightforward that the summand (5.5) is isomorphic to

$$
\operatorname{Hom}_{\mathcal{H}_{r}}\left(M^{\mu^{(1)}}, \mathcal{H}_{r}\right) \otimes_{R} \operatorname{Hom}_{\mathcal{H}_{r}}^{\wedge}\left(M^{\mu^{(2)}}, M^{\mu^{(1)}}\right) \otimes_{R} \cdots \otimes_{R} \operatorname{Hom}_{\mathcal{H}_{r}}^{\wedge}\left(M^{\lambda}, M^{\mu^{(k)}}\right),
$$

First, to show the correspondence of factors between $\widehat{B}_{k}^{\lambda}$ and $\widetilde{B}_{*}^{\lambda}$ in non-negative degrees, it is enough to give an isomorphism of $\mathcal{H}_{r}$-modules $\phi_{\nu}: \operatorname{Hom}_{\mathcal{H}_{r}}\left(M^{\nu}, \mathcal{H}_{r}\right) \rightarrow$ $\operatorname{Hom}_{R}\left(M^{\nu}, R\right)$, for every $\nu \in \Lambda(n, r)$.

With the natural anti-automorphism of $\chi: \mathcal{H}_{r} \rightarrow \mathcal{H}_{r}$ and $f \in \operatorname{Hom}_{\mathcal{H}_{r}}\left(M^{\nu}, \mathcal{H}_{r}\right)$, we can define right module structure of $\mathcal{H}_{r}$ on $\operatorname{Hom}_{\mathcal{H}_{r}}\left(M^{\nu}, \mathcal{H}_{r}\right)$ and $\operatorname{Hom}_{R}\left(M^{\mu}, R\right)$, which is given by the formula $(f \sigma)(m)=f(m \cdot \chi(\sigma))$, where $m \in M^{\nu}$, and $\sigma \in \mathcal{H}_{r}$.

For $f \in \operatorname{Hom}_{\mathcal{H}_{r}}\left(M^{\nu}, \mathcal{H}_{r}\right)$ and $m \in M^{\nu}$, define $\phi_{\nu}(f)(m)$ to be the coefficient of $T_{i d}$ in $f(m) \in \mathcal{H}_{r}$. Note that $\left\{T_{w} \mid w \in \mathfrak{S}_{r}\right\}$ is an $R$-basis of $\mathcal{H}_{r}$, now we claim that $\phi_{\nu}$ is a homomorphism of $\mathcal{H}_{r}$-modules.

First, we have that $\phi_{\nu}(f) \cdot T_{w}=\phi_{\nu}\left(f T_{w}\right)$ because:

(i) $\left(\phi_{\nu}(f) \cdot T_{w}\right)(m)=\phi_{\nu}(f)\left(m T_{w^{-1}}\right)=$ the coefficient of $T_{i d}$ in $f\left(m T_{w^{-1}}\right)$,

(ii) $\phi_{\nu}\left(f T_{w}\right)(m)=$ the coefficient of $T_{i d}$ in $\left(f \cdot T_{w}\right)(m)=$ the coefficient of $T_{i d}$ in $f\left(m T_{w^{-1}}\right)$.

Assume that $\phi_{\nu}(f)=0$, then the coefficient of $T_{i d}$ in $f(m)$ equals to 0 , for any $m \in M^{\nu}$. By induction on the length of elements of $\mathfrak{S}_{r}$, suppose that the coefficient of $T_{w}$ in $f(m)$ equals to 0 , for any $m \in M^{\nu}$ and $\ell(w) \leq n$.

Let $w^{\prime} \in \mathfrak{S}_{r}$ with $\ell\left(w^{\prime}\right)=n+1$. Using the Mathas's formula in [22], one can find $s=(i, i+1) \in \mathfrak{S}_{r}$ for some $1 \leq i \leq r-1$ such that

$$
T_{w^{\prime}} T_{s}=q T_{w^{\prime} s}+(q-1) T_{w^{\prime}}, \quad \text { and } \quad n=\ell\left(w^{\prime} s\right)<\ell\left(w^{\prime}\right)=n+1 .
$$


Meantime, $f(m) T_{s}=f\left(m T_{s}\right)$, and write $f(m)=\sum_{\ell(w)>n} \alpha_{w} T_{w}$ for some $\alpha_{w} \in R$. Then we have

$$
0=f\left(m T_{s}\right)=\sum_{\ell(w)>n} \alpha_{w} T_{w} \cdot T_{s}=q \alpha_{w} T_{w s}+\sum_{\substack{\ell(w) \geq n \\ w \neq w_{0} s}} \alpha_{w}^{\prime} T_{w} .
$$

It follows that $q \alpha_{w}=0$ then $\alpha_{w}=0$ since $q \neq 0$. Therefore, we find out that $f(m)=0$ for any $m \in M^{\nu}$ and then $f=0$, which implies the homomorphism $\phi_{\nu}$ is injective.

As free $R$-modules, it is obvious that the rank of $\operatorname{Hom}_{\mathcal{H}_{r}}\left(M^{\nu}, \mathcal{H}_{r}\right)$ equals to that of $\operatorname{Hom}_{R}\left(M^{\nu}, R\right)$, which means the homomorphism $\phi_{\nu}$ is surjective. It implies that $\phi_{\nu}$ is an isomorphism.

Lastly, by Definition 3.7, it is straightforward that the isomorphism $\phi_{\nu}$ indeed induces a chain map from $\widetilde{B}_{*}^{\lambda}$ onto $\widehat{B}_{*}^{\lambda}$, which is just the isomorphism from $\widetilde{B}_{*}^{\lambda}$ to $\widehat{B}_{*}^{\lambda}$.

Remark 5.4. With help of the above theorem and Theorem 4.7, we have shown that the Boltje-Maisch complex $\widetilde{B}_{*}^{\lambda}$ is exact for $\lambda \in \Lambda^{+}(n, r)$.

Acknowledgements: The authors thank the support from the National Natural Science Foundation of China (No.11271318, No.11171296 and No. J1210038) and the Specialized Research Fund for the Doctoral Program of Higher Education of China (No. 20110101110010) and the Zhejiang Provincial Natural Science Foundation of China (No.LZ13A010001).

\section{REFERENCES}

[1] K.Akin, On complexes relating the Jacobi-Trudi identity with the Bernstein-GelfandGelfand resolution. J. Algebra 117 (1988), 494-503.

[2] K.Akin, D.A.Buchsbaum, Characteristic-free representation theory of the general linear group. Adv. in Math. 58 (1985), 149-200.

[3] K.Akin, D.A.Buchsbaum, Characteristic-free representation theory of the general linear group II. Homological considerations. Adv. in Math. 72 (1988), 171-210.

[4] R.Boltje, R.Hartmann, Permutation resolutions for Specht modules. J. Algebraic Combin. 34 (2011), 141-162.

[5] R.Boltje, F.Maisch, Permutation resolutions for Specht modules of Hecke algebras. J. Algebra 365 (2012), 12-28. 
[6] R.Dipper, G.James, Representations of Hecke algebras of general linear groups, Proc. L.M.S (3), 52 (1986), 20-50.

[7] R.Dipper, G.James, A.Mathas, Cyclotomic q-Schur algebras, Math. Zeit., 229 (1999), 385416.

[8] S.Donkin, On Schur algebras and related algebras I, J. Algebra 104(1986), 310-328.

[9] S.R. Doty, Resolutions of B modules. Indag. Mathem. 5(3) (1994), 267-283.

[10] J.Du, H.B.Rui, Ariki-Koike Algebras with Semi-simple Bottoms. Math. Zeit. 2000, 204, 807-835.

[11] J.Du, B.Parshall and J.-p.Wang, Two-parameter quantum linear groups and the hyperbolic invariance of q-Schur algebras, J. London Math. Soc. 44 (1991), 420 C436.

[12] J.Du, H.B.Rui, Borel Type Subalgebras of the $q$-Schur ${ }^{m}$. Journal of Algebra, Volume 213, Issue 2, 15 March 1999, Pages 567-595

[13] J.Du, H.B.Rui, Specht modules for Ariki-Koike algebras, Comm. Algebra 29 (2001) 47104719.

[14] J.Du, L.Scott, Lusztig conjectures, old and new. I, J. Reine Angew Math. (1994).

[15] J.A. Green, On certain subalgebras of the Schur algebra. J. Algebra 131 (1990), 265-280.

[16] J.A.Green, Polynomial Representations of $G L_{n}$. Lecture Notes in Math., vol. 830, SpringerVerlag, Berlin (1980)

[17] W.van der Kallen. Longest weight vectors and excellent filtrations, Math. Z. 201(1989), 19-31.

[18] M.Kashiwara, Crystal base and Littelmann's refined Demazure character formula. Duke Math. J., 63 (1993), no. 3, 839-858.

[19] M. Kashiwara, On crystal bases of the $q$-analogue of universal enveloping algebras, Duke Math. J. 63 (1991), 465-516.

[20] G.Lusztig, Introduction to Quantum Groups, Birkhäuser, Boston/Basel/Berlin(1993).

[21] S. MacLane, Homology, Springer-Verlag, Berlin, 1963. Zentralblatt MATH: 0133.26502.

[22] A.Mathas, Iwahori-Hecke Algebras and Schur Algebras of the Symmetric Group. Univ. Lecture Ser., vol. 15 Amer. Math. Soc. (1999).

[23] S.Ryom-Hansen, A q-analogue of Kempf's vanishing theorem, Moscow Mathematical Journal Volume 3, Number 1, Pages 173-187.

[24] A.P.Santana, The Schur Algebra $S(B+)$ and Projective Resolutions of Weyl Modules. Journal of Algebra, Volume 161, Issue 2, November 1993, 480-504.

[25] A.P.Santana, I.Yudin, Characteristic-free resolutions of Weyl and Specht modules. Advances in Mathematics, Volume 229, Issue 4, 1 March 2012, 2578-2601

[26] D.J.Woodcock, Borel Schur algebras. Comm. Algebra 22 (1994), no. 5, 1703-1721.

[27] D.J.Woodcock, A Vanishing Theorem for Schur Modules. Journal of Algebra, Volume 165, Issue 2, 3 March 1994, Pages 483-506.

[28] D.J.Woodcock, Schur algebras and global bases: New proof of old vanishing theorems, J. Alg. 191 (1997), 331-370.

[29] D.J.Woodcock, Borel Schur algebras, Comm. Algebras 22(5)(1994). 1703-1721.

[30] I.Yudin, On projective resolutions of simple modules over the Borel subalgebra $S^{+}(n, r)$ of the Schur algebra $S(n, r)$ for $n=3$. J. Algebra 319 (2008), 1870-1902. 
[31] A. V. Zelevinski, Resolvants, dual pairs, and character formulas. Funct. Analysis and Its Appl. 21 (1987), 152-154.

\section{Xingyu Dai}

Center of Mathematical Sciences, Zhejiang University, Zhejiang 310027, China.

E-mail: daixingyu12@126.com

Fang Li

Center of Mathematical Sciences, Zhejiang University, Zhejiang 310027, China.

E-mail: fangli@zju.edu.cn

\section{Kefeng Liu}

Center of Mathematical Sciences, Zhejiang University, Zhejiang 310027, China.

Department of Mathematics, University of California, Los Angeles, USA

E-mail: liu@math.ucla.edu 